\begin{tabular}{llllllll} 
E & S & T & U & D & I & O & S \\
\hline
\end{tabular}

\title{
LA ODONTOPEDIATRÍA EN LA OBRA DE FRANCISCO MARTÍNEZ
}

\section{Martín Romero Maroto}

En marzo de 1557 se publica el primer libro español dedicado íntegramente a la odontología: El Coloquio breve y compendioso sobre la materia de la dentadura y maravillosa obra de la boca, cuyo autor es Francisco Martínez, nacido en Castrillo de Onielo (Palencia) hacia 1520 y fallecido en Alameda del Valle en 1585.

Francisco Martínez fue dentista de Felipe II y dedicó el libro al príncipe Carlos. Primero Bachiller y luego Licenciado, reeditó su obra en 1570.

Actualmente se conservan pocos ejemplares originales pero existe una edición facsímil promovida por D. Pedro García Gras en 1975.

Francisco Martínez se valió para construir sus discursos del diálogo. Valerio, caballero de buena familia, conversa con Ramiro, un antiguo criado afligido por la enfermedad de su hijo que sirve para desgranar lo relativo a la primera dentición.

Entre las fuentes que utiliza el autor hay que incluir autores contemporáneos como Montaña de Montserrat y Laguna y autores clásicos, citando Martínez entre otros a Aristóteles, Galeno, Cicerón, Platón, etc. pero todo esto queda superado por las propias experiencias personales que el autor aporta, algo propio del Renacimiento en cuya mentalidad lo que prima no es una actitud de ruptura con el pasado, sino la de lograr un mejor conocimiento de los clásicos a los que se venera. Lo que ocurre es que debido a la fiebre de experiencia personal, que también es propia de 
la época renacentista, surge muchas veces la evidencia de que la realidad difiere de las ideas que los clásicos legaron. Paradójicamente la veneración por la antigüedad clásica conduce a la ruptura con la ciencia antigua y a la instauración de una ciencia moderna.

Francisco Martínez escribió este libro porque consideraba que los temas bucales no eran tratados con el rigor y consideración que merecían en comparación con otras partes del ser humano, y lo dirigió al gran público pues la obra es fundamentalmente de divulgación, lo que no excluye que algunos profesionales, fundamentalmente los barberos, pudieran encontrar en ella una gran utilidad.

El libro toca fundamentalmente temas de encía y dientes, excluyendo otros temas estomatológicos frecuentes en la época como las úlceras o la ránula.

El libro fue plagiado por un barbero napolitano: Juan Bautista Xamarro, quien copió íntegramente la obra de Martínez, si bien esta copia nunca llegó a publicarse, aunque puede encontrarse el manuscrito en la Biblioteca Nacional de Madrid. Analizamos a continuación las ideas que sobre odontopediatría se vierten en este tratado.

\section{Anatomía}

Las primeras nociones sobre odontopediatría del coloquio aparecen para justificar la existencia de dos denticiones, algo que hacen otros autores de la época como el anatomista Montaña de Montserrat (1) en función de que los primeros dientes son muy tiernos y de que en la primera edad se comen cosas muy duras, por lo que es necesario el recambio dentario.

Francisco Martínez coincide con los anteriores autores, pero aporta además otras razones que ya enlazan más con lo que es la práctica estomatológica. Así, una de las razones por las que hay dos denticiones es porque como en la infancia las fracturas dentarias son muy frecuentes, con el recambio se evita que la secuela de estas fracturas sea duradera. Por otra parte; si los dientes de leche fueran muy fuertes, el recambio sería más difícil, con lo que tanto las maloclusiones por malposición dentaria, como las lesiones yatrogénicas que se producen en la extracción, serían más frecuentes.

Por otro lado, si los niños nacieran con dientes, la lactancia sería más difícil, pues dañarían el pecho de su madre. Pese a su clara visión prácti- 
ca de las cosas (no podemos olvidar que la indicación actual de extraer un diente neonatal es precisamente esa lesión que puede provocarse en el pecho materno, ni que las fracturas dentarias son evidentemente más frecuentes en los niños), hay un error importante en ese diagnóstico, cuando afirma que los dientes de leche no tiene raíces, lo que.indica que no debió extraer ninguna pieza temporal, si no fue ya muy cerca del momento de su exfoliación. Leamos sus texto:

«Lo primero porq al principio como todo el mantenimiento es la leche de la madre necessariamente la materia de los dientes auia de ser tierna y muy delicada y por consiguiete porque la madibula esta muy delgada y tierna, no se podia alli bien formar ni conseuar rayz y aun por tercera que no me dexa de quadrar, y es que los niños desde q se comiençan a saltar hasta la edad de siete $u$ ocho años, $q$ es quando comiençan a mudar dan muchos golpes y caydas, y si acertase a quebrarsele algun diente, qdaua sin remedio; $d$ aquella edad adelante tiene mas fuerça para tenerse y no caer, y mas sentido para mirar por si» (2).

"Forma naturaleza la boca del hombre al principio y nascimiento sin dientes hasta cierta edad,-no sin causa y razon. Porque tener los niños recien nascidos detadura no les conuenia porq no tienen fuerça en la boca para vsar dellos, ni calor en el estomago para digerir lo que co ellos exercitasen, antes dañarian a sus madres y lastimarian los pechos, y ansi mismos se estouarian para el vso del paladear.

Ya q los niños van cresciedo y tomado calor en el estomago, para poder digerir algunos majares, y fuerça en la boca para poder exercitar los dietes, les proueyo de vnos dietecillos tiernos y sin rayces, tiernos segu sus fuerzas y calor del estomago sin rayces porque han de mudarlos y trocallos muy psto por otros. Y si estos primeros nascieran con rayces vuiera tres inconuenietes. El primero que no se mouieran tan facilmente quando viniera los otros nueuos. El segundo que los q vinieran hallaran los vasos occupados y salieran tuertos, como acontesce aora muchas vezes por no tener especial cuydado de sacarselos co tiempo. El tercero la difficultad que vuiara de sacarselos, que por estar tierna la mandibula se rasgara como aora algunas vezes acotese sacando muela o diente que tenga rayz y en tal caso nunca el diente q viniera fuera muy firme ni duradero, por la falta y flaqueza de la madibula» (3).

En este período los conocimientos sobre el recambio dentario e incluso sobre el número de denticiones parecen estar muy confusos. Evidentemente se conoce la caída de los dientes, pero pocos autores hablan de la 
dentición temporal, ni sobre el orden del recambio, si exceptuamos a Francisco Martínez que parece tener muy claro cuando se completa la erupción de los temporales y'cuando se produce su exfoliación:

«Desde los dos años o dos y medio que nasce hasta los siete u ocho que se mudan» (4).

Y aún afina más:

«La edad de siete u ocho años es quando comiençan a mudar» (5).

Pero en realidad, no se conoce aún el momento ni el orden de erupción de cada pieza, qué piezas necesitan que para erupcionar caiga un diente deciduo y cuáles no, en definitiva, se puede concluir que durante los siglos XV y XvI, el recambio dentario no es un tema bien conocido, pues incluso Francisco Martínez no habla expresamente de esos temas y en un texto en el que muestra su espíritu científico, deja abierta la duda:

«Me ha certificado y certifica, q ay hombre q cada vn año muda la detadura, crealo quie quisiere o no lo crea, que yo no lo osara affirmar, ni aun dezir sino viniera ta approposito. $Y$ assi hare de todo lo de mas que no vea, y sepa de cierta sciencia, aunq pueda acontescer naturalmente» (6).

Aunque el hecho de que sea el autor que más relación práctica tuvo con el arte dental le permitió observar fenómenos que otros autores ni imaginaron, como por ejemplo, el hecho de que un molar temporal no se exfolie y dure muchos años, llegando en su perspicacia a intuir la agenesia. Curiosamente en este caso, sí afirma que el molar temporal tenía raíces, pero lo ve como un hecho excepcional, justificado precisamente por la agenesia:

"Yo he visto por muchas vezes hombres que nunca mudaro alguna muela, y esta rezia, y con sus rayzes. Parecesme a mi que lo que es de aquella tal muela pudiera ser de toda la detadura y ansi no tuuiera nescesidad de mudarse. Es verdad porq naturaleza preuiene muchas vezes en 
lo que despues ha d faltar, como alli no auia de nacer otra, proueyo de darla aquella rayz. Mas esto ha lugar y acontesce solamente en las muelas por estar mas gruesa y acha la mandibula, que no en los dietes, y ansi en la parte de los dientes nunca se haze, y si acaso acontesce dos cosas te podre dezir: La primera, que vna golondrina no haze verano. La seguda, que estos tales dietes y aun las muelas q no se mudan no son perfectos ni durables y hasta en el color son differentes de los otros» (7).

En los textos de esta época surge frecuentemente la idea de que el proceso eruptivo es contínuo a lo largo de toda la vida. Esta idea, defendida por Juan Valverde de Hamusco (8) y Dionisio Daza (9), tiene su origen en Galeno y siguió siendo defendida por Vesalio (10).

Sin embargo, Francisco Martínez mantiene la opinión contraria: los dientes erupcionan hasta un determinado momento y a partir de ahí, ya no hay más erupción:

«Despues de mudados los dietes y muelas crecen hasta cierto tiempo y no mas y de aqui viene el engaño $d$ algunos que dizen que los dientes disminuye harto contrario de otros que dize que crecen toda la vida.

Los dientes en tiniendo el tamaño que han de tener se les acaba la virtud que llama los medicos formatiua y dexan de crecer. De aqui nasce el principio de vn engaño que como los dientes crescen todo lo que han de crecer mucho antes que todos los miebros parescen grandes, por ser el cuerpo donde estan pequeño y despues como todos los otros miebros crecen y los dietes no, proporcionanse los dientes con el cuerpo y con todas las partes del, y assi quado era pequeño parescian grades, por razo que vno esta en proporcion de lo otro, pero no por esto diminuyeron" (11).

Las dos opiniones están bastante cercanas a las ideas actuales sobre erupción. Es cierto, como defiende la mayoría de los autores, que el potencial eruptivo no se agota con la edad, y si no se manifiesta es porque los factores que determinan el equilibrio estomatognático permiten que se mantenga una posición estable. Pero no es menos cierto, y ahí enlazan mejor las ideas de Francisco Martínez, que, antes de llegar a este equilibrio, hay una fase de erupción activa que ocurre en la fase puberal entre los trece y los dieciocho años, y en la que el desarrollo facial condiciona una adaptación dentaria que los dientes consiguen con una erupción ac- 
tiva de dos o tres milímetros. Cuando se acaba el crecimiento facial (18 ó 20 años) se alcanza un nuevo equilibrio eruptivo y las piezas dentarias alcanzan su destino final (12).

No estoy afirmando que Francisco Martínez conociera hasta este punto los mecanismos de erupción, pero es evidente que estas ideas vienen inmediatamente a la mente cuando uno lee sus textos.

\section{Patología médica}

Francisco Martínez apenạs si toca en su libro los temas que en el momento eran estudiados por médicos o cirujanos, intentando limitarse a las afecciones dentales.

En esa línea no habla para nada en su obra de temas como las aftas, las fisuras labiales, la ránula, o los frenillos, citando de forma somera la patología que la erupción de las pienzas dentarias ocasionaba, tema ampliamente estudiado en la obra de otros autores contemporáneos suyos. Se limita a recomendar que no se usen en la infancia medicinas violentas y sugiere que se laven las encías con vino blanco si el humor responsable es frío y con agua de Llanten o agua de cabezas de rosas si es caliente (13).

\section{Extracción dentaria}

El tema de la extracción dentaria es posiblemente el que más directamente se relaciona con la práctica odontológica de la época; ésa es la razón por la que Francisco Martínez es el autor que realmente trata el tema, pues sus textos son, junto con una breve cita de Dionisio Daza en la que recomienda que se extraiga el diente temporal si el definitivo erupciona en mala posición, los únicos que hablan de este tema refiriéndose a los niños.

Francisco Martínez, en los diversos avisos que da para lo que él llama la segunda disposición de los dientes y que comprende el período que va desde la erupción de los temporales hasta su caída (14); cubre el diagnóstico; el instrumental necesario, las indicaciones y las complicaciones de las extracciones en la dentición temporal.

Hay que destacar que cuando habla de extraer piezas temporales, resalta que es bueno hacerlo "si no tienen cura" y es que esa frase ya deja 
clara una mentalidad conservadora respecto a las piezas temporales, que ni siquiera han tenido muchos odontólogos de siglos posteriores, incluido tristemente el siglo xx. Pues bien, cuando estas piezas ya son incurables, Francisco Martínez piensa que es mejor extraerlas para evitar el dolor que se produce cuando se afecta la pulpa, para evitar la extensión del proceso careoso a otras piezas y para evitar la halitosis.

El desconocimiento que sobre erupción se tiene en la época, le hace alabar el que el resto de dientes se junten, cerrando el hueco del diente perdido. Es claro que con esta mentalidad, muchas piezas definitivas quedarían incluidas en los maxilares.

"Si no tiene cura se ha de sacar antes q se acabe de comer, porq se euitan tres daños y se hazen vno de los prouechos. El primer daño es el dolor $\mathrm{q}$ tiene $\mathrm{o}$ ha de tener forçosamente llegando la corrupcion al neruezillo o venas del diente. El segundo que estoruaran q no se pegue a las otras sus vecinas. El tercero que no abra el mal olor en la boca que de tener aquel raygon o muela podrida se ha de feguir. El vno de los bienes sera, o que no nacera otro donde se sacare aquella, si la virtud formatiua no es acauada como acostece muchas vezes en esta edad o, ya que esto no sea vernase a juntar tato las unas con las otras que casi no echara de ver su falta. Tabie como la mandibula todavia en esta edad crezca y resciua virtud de nueuo, viene a apretarse en si tanto con el diente o dientes que queda, que los dexa rezios y fuertes» (15).

Cuando intenta ver en qué muela está el problema, utiliza como medio diagnóstico la percusión y el escarbar en la caries para ver cual es más profunda. Aporta además dos posibles métodos diagnósticos cuando la caries es interproximal:

«El nono auiso es, que muela se ha d sacar estando en duda qual duele. Porque acontesce muchas vezes sacar la buena y dexar la mala, y que haze el dolor: Lo primero que se ha de mirar: si ay alguna dañada o no. Si no esta dañada ninguna, han de tomar vn hierro que tenga vna cabeçita como este q esta aqui pintado, y dar en las muelas de que se tiene sospecha, y en la que mas se sintiere el dolor esta el mal y se ha de sacar, y si ay alguna'corropida mirar si es solo vna o mas, si no ay mas de vna manifiesto es que esta alli el daño; y que aquella se deue sacar. Si estan dañadas mas que vna, han de tomar un hierro que tega una puta a manera de lancilla como aqui va pintado y d la otra parte una buelta como garauatillo 
para las muelas que se comen por detras y co el escauar en lo podrido y despues de quitado lo malo en la q doliere mucho llegando el hierro esta el daño y causa del dolor, y es la que se ha $\mathrm{d}$ sacar. Esto esta claro porque si aqlla siete. el hierro mas que las otras, es porq esta mas comida, y mas descubierto el neruezillo y mas flaca, y como a tal se haze mas a ella que a las otras el corrimiento de reumas.

Y porque muchas vezces acontesce estar el daño etre muela y muela, $\mathrm{q}$ no se puede ver, en tal caso ha de hazer la prueua del hierro que tiene la cabeçita dado en cada vna de las muelas que se tiene sospecha, y lo demas como tengo dicho.

Tambie se puede apartar la una de la otra y hazer la experiecia del hierro de la puta. Pero todo esto quiere gra cuydado subtileza y experiencia. Ase de apartar la muela con vn hierro a manera de escoplito muy afilado, y con vn martillico, porque se haze mas facil y delicadamente, y mas sin pesadumbre que con lima aunque podria acontescer alguna vez que vuiesse necessidad de limar algo para que se cortase mejor" (16).

En cuanto al instrumental, marca claramente que es lo que debe usarse según la situación particular de la pieza a extraer, explicando claramente cuando deben usarse los instrumentos con los que Paré había enriquecido el armamentario odontológico: El gatillo y el pelícano (17).

«El dezimo auiso es para que sepan quando se ha de sacar la muela con gatillo, y quando con policano. Porque acontesce muchas vezes, qbrar la muela por no lo faber. Quando la muela no esta corrompida o esta poco comida que pueda sufrir alguna fuerça sin quebrarse, ase $\mathrm{d}$ facar con gatillo, porque se saca mas facil y presto, y sin pesadumbre de la otra dentadura que queda. Pero si la muela se tiene alguna sospecha q se ha de quebrar, hase de sacar con polican: Porque si le sabe bien exercitar aseguran la muela, aunque este mas podrida que no se descabeçe. Quando hay algún pedacito de muela o raygon que se ande hase de quitar co una destas dos herramientas que tengan por la parte que asen vna raytas menudas y hodillas como estas. Quando en las muelas postreras acontesciere tener algunas puntillas q den pesadumbre o gasten sus opuestas han se de cortar co vn hierro como este muy afilado, y co el martillico" (18).

Aparte de la caries muy profunda, la otra indicación que_da para la extracción de las piezas temporales, es permitir él buen alineamiento de las piezas permanentes, lo cual se consigue según él, extrayendo antes de su erupción las piezas deciduas. 
«El qrto de q se deue tener gra cueta es en sacarlos con tiepo especial si son dientes porque los nueuos que vienen salgan bien, y no hallen ocupados los vasos y lugares por donde han de salir con los viejos» (19).

«Ha de tenerse la cueta de sacarlos co tiepo antes que los otros salgan. En las muelas no es tanto inconueniente, porq como son mas anchas y gruesas que los dietes, siempre la que viene toma a la vieja por medio y la leuanta derecho, y sale ella bie lo q no hazen los dietes. Porque como son angostos los que estan y puntiagudos los que vienen facilmente se pueden torcer a una y otra parte y de aqui es que en las muelas no hay otro inconueniente sino que podrian pegar a las otras que viene el neguijon» (20).

Dos ideas están latentes en estos textos: por un lado, la posibilidad de que un diente temporal careado afecte a su sucesor y aunque, evidentemente, el autor no habla de las hipoplasias de esmalte, que en los dientes permanentes pueden producirse por focos infecciosos largamente mantenidos en procesos careosos de los temporales, parece que de alguna manera lo inituye. Por otro lado, el tema del espacio libre, o sea, la diferencia de tamaño entre los dientes primarios y sus sucesores permanentes, que en el sector anterior es negativo y en el sector posterior positivo (21), lo que causa que el apiñamiento sea más frecuente en el sector anterior. Está claro que Francisco Martínez observa este fenómeno, aunque lógicamente, su intento de explicarlo en función de la diferente morfología entre dientes y muelas, no sea exacto.

Tiene claro en cualquier caso, que esta extracción con fines de alineamiento dentario, sólo debe hacerse cuando ya la exfoliación es inminente, destacando de nuevo la importancia de que las piezas temporales se mantengan hasta ese momento. Esta idea suya puede justificar su error de considerar que los molares temporales no tienen raíces: Su espíritu conservador lo llevó a desconocer esa faceta de la anatomía dentaria; así, cuando se le plantea el tema de extraer pronto las piezas temporales, responde:

«No tampoco, porque la mucha diligencia suele dañar, ellos'dan luego señal, quando vienen los otros en que se andan y si mirays en ello, mas lastima es que le falten presto, quanto mas que como los dientes antes que se muden no tienen rayces, no es nada el dolor, ni difficultoso sacarlos y puedese vsar desta cautela: Quado se comiença a andar el diete atarle un hilo antenoche, diziendo que es para otra cosa, y quando durme tirar dl, saldra que apenas le despierten" (22). 
Entre los problemas que pueden aparecer al extraer las piezas a los niños, uno de ellos es puramente yatrogénico: confundir la pieza temporal con la pieza permanente. En estos casos, además de alertar para que se eviten errores, explica como debe hacerse la extracción, en función de hacia donde se coloque el temporal.

«El septimo es para los barberos y maestros de sacar muelas que tega vigilancia y cueta en vna cosa que mucha va y la miran poco. Acontesce nacer vn diete y quedarse el viejo tambie, y como el vno dellos se ha de sacar por fuerça, por superfluo o ser feo e tal caso los q viene a sacar algun diete de aquellos no miran mas de al buen parescer y assi facan el mas tuerto. Esto es muy gran error porq si aciertan a sacar el nueuo y dexar el viejo, quedaran muy presto sin el vno y sin el otro: Porque aquel tal que dexan no tiene rayces, y assi cae y peresce muy presto. De manera que ha de sacar el viejo y despues sus padres se le llegaran poco a poco a su lugar, e coprimiendole hazia donde ha de estar, que como esta ternezilla la mandibula se porna muy facilmete en su lugar, esto se ha de hazer muy despacio, cada dia quatro o cinco vezes hasta que este en su asșieto y buen orden con los otros y todo esto se escusara con tener cuenta de sacallos con tiempo antes que salga el nueuo torcido.

Estos dentecillos se ha d sacar con vn botador como este. Si el diete esta de parte de fuera, co la buelta del hierro, y si de parte de dentro con la punta derecha. Pero si acotesciere salir'algu diete muy dentro de la boca hanle de sacar con vn botador como este y co esta buelta: Porque si la mano se demandare con la buelta se detenga afirmando en los dietes y no lastime en la garganta o en otra parte alguna de la boca» (23).

Notamos en este texto cómo el autor es consciente de que una vez que el diente en su proceso eruptivo emerge en la cavidad bucal, puede ser movido por estructuras anatómicas (labio, carrillo, lengua) o por objetos extraños que se lleven a la boca, como los dedos de los padres; es el inicio de un movimiento ortodóncico algo rudimentario.

El segundo problema es la hemorragia post-extracción para la cual recomienda que en los niños sólo se use vitriolo si es grave, pero si no, se use esponja quemada o pelos de liebre.

«El octauo auiso es, que quando por sacar alguna muela vuiere fluxo de sangre, como muchas vezes acontesce: En tal caso ha de tomar vitriolo romano molido, y si quisiere quemado, y hazer vnas peloticas de hilas a 
manera de garbanços pequeños y cargallas de aquellos poluos y ponellas en el vaso por donde sale la sangre, lauandola primero co vn poco de vino cozido con alumbre, encieso y mirra, y vn poco de romero, y espicanardi, y si el fluxo es poco podra teplar el vitriolo co mezclar con ello vn poco de bolarmenico. Este remedio del vitriolo no se deue vsar en la primera y segunda edad, por ser caustico y medicina fuerte, sino fuesse en caso de mucha necessidad. En lugar de vitriolo podran poner poluos desponja qmada; o pelos de liebre, y cosas ansi, lauandolo primero con el vino que tengo dicho» (24).

La hemorragia post-extracción era curiosamente defendida por Paré, con el fin de que pudieran eliminarse los humores mórbidos. Paré la controlaba más tarde entre otras cosas, con la presión. Esta defensa de la hemorragia post-extracción, que enlaza claramente con la medicina humoralista, muestra la influencia que sobre Paré ejerció Galeno, autor a quien Paré leyó e incluyo tradujo (25).

\section{Odontología conservadora}

Al entrar en la odontología conservadora hay que definir lo que para los autores de la época es el neguijón, que en realidad es lo que hoy llamamos caries.

El neguijón se considera una alteración humoral que pudre y ennegrece el diente, si bien aún quedan autores que atribuyen la etiología del proceso a los gusanos.

Es lógico que la etiología de la caries se oriente desde una doble vertiente humoral y vermicular, porque todavía no se habían observado gérmenes en las lesiones y por tanto aún no había comenzado a pensarse en los gérmenes como agentes causales de la caries.

En cuanto al neguijón infantil, parece ser frecuente si atendemos a los textos de Francisco Martínez.

«En esta edad y dispusicio dos cosas son las mas ordinarias: Apostemarse la enzias y:corromperse los dietes que es el neguijo que llaman» (26).

Este autor, con gran intuición, otorga al factor hereditario un papel en la causa de la enfermedad: 
«A su padre paresce en vna cosa, que me pesa dello. En' la dentadura ya se le comiençan a corroper los dietes» (27).

Concede gran importancia a la afectación de los dientes de leche, y así, en su libro, escrito en forma de coloquio, cuando Cristolo comenta que no es gran inconveniente que los dientes se corrompan antes de mudarse, Valerio le contesta que sí lo es y muy importante, si no se remedia (28).

La importancia que le otorga está en función de que pueden afectar a sus sucesores, que pueden afectar a las estructuras óseas que les sirven de soporte, y de que, al no ver cuando se mueven y no extraerlos con tiempo, pueden ocasionar malposiciones dentarias. Hace así referencia a las grandes pérdidas de espacio que se producen por las grandes caries en dientes temporales y que originan malposiciones dentarias, aunque él lo achaque a no haber hecho la extracción a tiempo:

«Si se corrompe los dientezillos, han de procurar de atajar que no passe adelante la corrupcion, porque de comerse de neguijon antes que se mude ay dos inconuenientes. El primero q quado esta comidos y que no hay mas que las rayzes no se echa de ver quando se andan y es tiempo de sacallos. El segundo porque son peores de sacar, y destos dos inconuenientes nacen tres daños. El vno que estos q estan por mudar y comidos de neguijon lo podrian pegar a los que vienen porque con su corrupcio estragan y corropen la complexion substancia y mantenimiento de la mandibula y partes circunstantes. El otro que de no sacarse los viejos co tiempo los nueuos que ha de venir, como halla ocupados los vasillos por donde han de salir, vienen a nacer tuertos y d mala manera como saldria y sale vna heruecilla en el capo si topa con alguna piedra, o otro impedimento que no la dexa brotar por derecho y deste daño de no salir bien los dientes nacen otros grades. El primero, el mal parescer q no es pequeña fealdad tener mala postura de dientes. El segundo, q no son duraderos: Porque vnos con otros se vienen a mover y echar fuera por tocarse de cuesta y no de puta: Como vemos en vn madero, que si esta hincado en tierra y bien puesto aploma por mucha carga q tenga ni golpes que le den encima, no se mueue a vna ni otra parte: Pero si esta mal puesto o trastornado ni substenta lo vno ni sufre lo otro. Assi hazen los dietes por esta razo qualquier cosa que se haga de fuerça en ellos les haze detrimeto» (29). 
En cuanto al tratamiento, Francisco Martínez es partidario de tratar el neguijón cuanto antes, y esto, en función de que se da cuenta que debido a la estructura dental, la enfermedad progresa lentamente al principio y más rapidamente después. Aunque él no sea consciente, está ya adelantando uno de los factores que diferencia al esmalte de la dentina: la dureza.

«La razon es esta, que el diente ni muela no son de vna misma materia ni condicion por la parte exterior que por la interior, porque no ay azero mas duro y rezio q es la primera camisa de la dentadura, ni madera mas blanda q ellos estan de dentro y ansi aquella primera camisa tarda mucho e corromperse pero pasada aqlla vase por la posta, como no halla resistencia la corrupcion de alli adelante y por esto se cura co facilidad al principio lo que no haze quado esta muy corrompido» (30).

En cuanto a la técnica, considera que dentro de la dentición temporal, son más fáciles de tratar los dientes que las muelas y nos describe como deben realizarse los tratamientos:

«El tercer auiso mirar si se corrompen los dentezillos en el punto que se les hagan vnas manchitas quitallas con vna herramienta muy afilada, que tega a la vna parte vna puta como laçuela y a la otra llanita como escoplito. Ase de vsar de cada parte deste hierro como fuere menester segun que fuere lo corrompido y despues co vn palillo mojado en un poco de vino cozido con vn poco de alumbre y sal comun darle en aquellas manchitas donde se quito lo podrido y si esto se haze con tiepo se remediara facilmente. En las muelas es mas difficultoso el remedio pero menos el peligro» (31).

Dentro de las divisiones que él establece según el proceso evolutivo de la boca, hay dos que caen de lleno en el campo de la odontopediatria: la primera es la que va desde los dos o dos años y medio que nacen los. dientes, hasta los siete u ocho, en que se caen; y la segunda, la que va desde que se mudan hasta los diecisiete o dieciocho años en que dejan de crecer (32), si bien ésta sólo en parte. Y es que, aunque él pueda analizar los períodos de dentición temporal y de dentición permanente, no anda tan fino cuando se refiere al período de dentición mixta. 
En cualquier caso, queda claro que el remedio que propone para conservar las piezas temporales es el que anteriormente hemos descrito y que en el período de dentición temporal se opone a medidas más agresivas, por los perjuicios que pueden causar.

\begin{abstract}
«Es.menester vn auiso necessario que ni que las enzias se aposteme ni los dietes se corrompan antes que se muden, no se ha de curar con medicinas violetas ni rezias ni cauterios de fuego, ni causticos, ni cosas desta manera: Porque sin comparacio seria mas el daño q el beneficio por estar la madibula tierna qualquier cosa le impediria que no rescibiesse virtud y quedara blaca y los dietes seran de poca dura» (33).
\end{abstract}

Sin embargo en la segunda disposición, es decir a partir de los siete años o período de dentición mixta, aunque también se muestra partidario de usar remedios suaves, acepta que se tomen medidas más agresivas si las piezas están muy afectadas:

«Se ha d mirar en esta segunda edad, si el diente se corrope, que se ha de curar tambien facilmente: Porque tapoco se sufre aqui medicinas muy rezias a causa que aunque la madibula y dietes tengan mas fuerça, aun no tienen toda la virtud que ha de tener y podria ser enflaquescerse. Ansi que en esta edad tabien se han de vsar medicinas liuianas mas ase de tener auiso, q si el inal va adelante, y no se puede sustentar con medicinas leues, que se vsen de las violetas y necessarias por dos razones: La vna porq ya e esta edad y seguda dispusicio el diete q se pierde pocas vezes se cobra, la otra es porq al principio remediados los males curase facilmete especialmete el neguijo q es muy leue de curar al principio y difficultoso si vna vez se apodera» (34).

Y más adelante recalca esta idea:

"A necessidad tabien sangra y purgan, cauterizan y sajan y hazen otras cosas a vn niño. Y vemos que todo esto le enflaquece y quita la virtud, mas al fin acuden a los mas necessario y de dos males escogen el menor. 
Si buenamete se puede pasar en esta seguda edad y dispusicio co medicinas leues que se escusen las mas fuertes por las razones dichas: Pero si se espera peligro que se usen violentas causticos y cauterios y todo lo demas necessario: Que mas vale q qde vn poco flaca la detadura que no que se pierda" (35).

Toca también Francisco Martínez, el tema de los flemones, para lo que da varios remedios incluyendo la apertura quirúrgica:

«El segudo mirar si se haze algun flemon ponerle un higo paso, para que se madure y abra, y si la materia fuere muy caliete poner vn poco de açucar rosada y si el flemo o apostema fuere tal que no baste esto, haran el cozimiento siguiente. Tomar passas sin granos, datiles sin cuescos, higos pasos açufayfas y ceuada, todo esto cozido en seys escudillas de agua o de caldo de cabeças de carnero q es mejor, o sino del mismo carnero. Ha de cozer hasta q gaste las dos escudillas y a este tiempo echar dentro violetas y oregano, y co esto torne a heruir vn poco, y despues colallo y esprimillo mucho, y exaguarse co ello, y este muy caliete quanto se pueda sufrir y tenerlo buen rato en la boca. Ỳ si fuere niño han de lauar con vn hisopico: Esto se haga muchas vezes hasta q se acabe el agua, o se abra el apostema y aun despues de abierto tabie aprouecha algunos dias, porq limpia mucho, pero quado vaya sanando podralo lauar co lo siguiete: Cozer vn poco de ceuada tostada y vn grano de alumbre e agua y colallo y echar vn poco de miel rosada y enxaguarse o lauarse con ello. Pero si con todo lo q he dicho, el flemon no se abriere sino q sea necessidad abrille con laceta $\mathrm{u}$ otro instrumeto qualquiera hase de tener cuenta de rasgar y abrirle bien. Porque si esto no se haze no se puede lauar ni limpiar bien y podrase venir a hazer sistola por la psencia del mal humor y carne podrida que ahoda y come la enzia y porque muchas vezes se hazen vnos flemocillos pequeños, y no ha menester tanto negocio como hemos dicho, puede hazer el cozimiento siguiete: Ceuada, rosas, violetas, pasas, higos, en la quatidad que les paresciere para media açumbre de agua o tres quartillos, ha d cozer que gaste la terzia parte y con esto caliete, quanto se puede sufrir enxuagarse o lauarse» (36).

Si bien el párrafo anterior se refería a la "primera disposición» (desde los dos años y medio hasta los siete u ocho) el tratamiento que propone para la "segunda disposición" (desde los siete u ocho años hasta los diecisiete) es el mismo: 
«El segudo que se ha de mirar si se haze algu flemoncillo. en esto me remito a lo pasado porque lo que cerca dllo dixe en la primera edad, se ha de entender para todas las demas» (37):

\section{Odontología preventiva}

Francisco Martínez habla ya de prevenir el neguijón y a este fin propone una serie de medidas dietéticas y métodos de higiene, pero estas medidas no van referidas específicamente a los niños.

En cuanto a la prevención de problemas periodontales, Francisco Martínez hace ya referencia a esto desde la primera edad (entre dos y siete años), cuando recomienda que los niñ்os coman con los dos lados, porque en el lado con que no se come se crían tovas que corrompen las encías (38).

De la misma forma se refiere a la segunda edad (entre siete y diecisiete años), aconsejando quitar la tova con mondadientes de tea o lentisco si es blanda y si es más dura de plata, oro o hierro. Los daños que según el autor provoca la tova son cinco: Gasta las encías, las enflaquece, aunque se quite ya es fácil que vuelva a aparecer, favorece que se corrompan los dientes y provoca halitosis (39).

La tova se corresponde con lo que hoy llamamos sarro. Julio González Iglesias nos explica el origen de estas dos palabras y como se relacionan en función de una antigua creencia sobre sủ formación:

«El nombre de toba proviene de una variedad de la caliza (cal carbonatada romboédrica) que junto con las pisolitas y trabertinos, tiene el mismo origen que las estalactitas y las estalagmitas, esto es disuelta en el agua se va depositando poco à poco por sedimentación. Nótese como ese mismo origen tiene la palabra tártaro; también sinónimo de sarro, sal que se deposita en los toneles de vino.

Ambos demuestran la antigua creencia de que el sarro se formaba por cierto depósito de sustancias disueltas en la săliva sobre la superficie de los dientes» (40).

En definitiva, este texto es no sólo el primer libro odontológico español, sino que supone también el primer texto que se preocupa de la dentición temporal, a la que da una gran importancia, explicando con detalle 
los tratamientos que en ella pueden realizarse. Podríamos pues considerar este libro como la cuna de la odontopediatría española, en un momento en que no sólo no existen las especialidades odontológicas, sino en que ni siquiera la odontología tiene entidad propia.

Escrito de una forma popular, mediante el diálogo, este texto debió ayudar a muchos barberos de la época a tratar un poco mejor a los niños.

Destacar estos hechos ha sido el objetivo de este trabajo y en definitiva su razón de ser.

\section{NOTAS}

(1) Montaña de Montserrat, B. (10), p. 36.

(2) Martínez, F. (9), pp. 33-34. La redacción de la Revista ha cambiado la grafía original de la letra $\mathrm{f}$ por la actual de s, con objeto de facilitar la lectura del texto: ser en lugar de fer, etc.

(3) Martínez, F. (9), pp. 83-84.

(4) MartíneZ, F. (9), p. 134.

(5) MartíneZ, F. (9), pp. 33-34.

(6) MartíneZ, F. (9), p. 93.

(7) Martínez, F. (9), pp. 86-87.

(8) Valverde de Hamusco, J. (14), p. 9.

(9) Daza Chacón, D. (6), p. 341.

(10) RING, M.E. (12), p. 121.

(11) MaRTíneZ, F. (9), pp. 88-92.

(12) Canut Brusola, J. A. (3), p. 27.

(13) Martínez, F. (9), p. 106.

(14) Martínez, F. (9), p. 101.

(15) Martínez, F. (9), pp. 128-129.

(16) MartíneZ, F. (9), pp. 114-117.

(17) Aroues Miarnau, R. (2), p. 108.

(18) Martínez, F. (9), pp. 117-119.

(19) MartíneZ, F. (9), pp. 45-46.

(20) MartíneZ, F. (9), pp. 104-105.

(21) Moyers, R. E. (11), p. 206.

(22) Martínez, F. (9), pp. 45-46.

(23) MartíneZ, F. (9), pp. 111-113.

(24) Martínez, F. (9), pp. 113-114.

(25) RING, M.E. (12), p. 129.

(26) Martínez, F. (9), p. 103.

(27) Martínez, F. (9), p. 99.

(28) Martínez, F. (9), p. 99.

(29) Martínez, F. (9), pp. 103-104

(30) Martínez, F. (9), pp. 126-127. 
(31) MartíneZ, F. (9), pp. 105-109.

(32) MarTíneZ, F. (9), p. 134.

(33) MarTíneZ, F. (9), p. 105.

(34) MartíneZ, F. (9), pp. 125-126.

(35) MaRTíneZ, F. (9), pp. 127-128.

(36) MARTÍNeZ, F. (9), pp. 106-108.

(37) MarTíNeZ, F. (9); pp. 123.

(38) MARTíneZ, F. (9), pp. 110.

(39) MaRTíneZ, F. (9), pp. 124-125.

(40) GonZález Iglesias, J. (8), p. 20.

\section{BIBLIOGRAFÍA}

(1) ALBERTI LÓPEZ, L. (1948). La anatomía y los anatomistas españoles del Renacimiento. Madrid, C.S.I.C.

(2) Aroues Miarnau, R. (1945). Historia anecdótica de la odontología. Barcelona, Salvat Editores.

(3) Canut Brusola, J.A. (1988). Ortodoncia clínica. Barcelona, Salvat Editores.

(4) Carmona Arroyo, F. (1972). «La literatura odontológica en el Renacimiento español». Medicina Española, 68, 400: 39-48.

(5) Carreras Panchón, A. (1974). «Las actividades de los barberos durante los siglos XVI al XVIII". Cuadernos de Historia de la Medicina Española, XIII: 205-218. Salamanca.

(6) Daza Chacón, D. (1584). Práctica y teórica de cirugía. Valladolid.

(7) Escribano GaRcía, V. (1934). La cirugía y los cirujanos españoles del siglo XVI. Granada, Tip. Lit. Paulino V.

(8) GonzÁlez Iglesias, J. (1981). El pasado de la higiene bucodentaria en España, Madrid. El mismo autor (1994) ha publicado, ya en prensa este trabajo, Historia de la Odontoestomatología Española, Madrid, Ediciones Avances. En él dedica un capítulo a nuestro autor.

(9) Martinez, F. (1557). Coloquio breve y compendioso sobre la materia de la dentadura y maravillosa obra de la boca. Valladolid.

(10) Montaña DE MonTserRat, B. (1550). Libro de la Anatomía del hombre. Valladolid.

(11) Moyers, R.E. (s.f.). Manual de Ortodoncia para el estudiante y el odontólogo en general, Buenos Aires, Ed. Mundi.

(12) RING, M.E. (1989). Historia ilustrada de la odontología. Barcelona, Ed. Doyma.

(13) Sánchez Granjel, L. (1968). Cirugía española del Renacimiento. Universidad de Salamanca.

(14) Valverde de Hamusco, J. (1556). Historia de la composición del cuerpo humano. 'Roma. 